\title{
A STUDY ON AUTOMATIC UAV IMAGE MOSAIC METHOD FOR PAROXYSMAL DISASTER
}

\author{
Ming $\mathrm{Li}^{\mathrm{a} *}$, Deren $\mathrm{Li}^{\mathrm{a}}$, Dengke Fan ${ }^{\mathrm{b}}$ \\ ${ }^{a}$ School of Remote Sensing and Information Engineering, Wuhan University, \\ NO.129, Luoyu Road, Wuhan 430079 \\ ${ }^{\mathrm{b}}$ The 3rd Railway Survey \& Design Institute, NO.10, Zhongshan Road, Tianjin 300142
}

KEY WORDS: Disaster Information, Image Mosaic, Image Matching, Bundle Adjustment, Dynamic Programming, Registration

\begin{abstract}
:
As everyone knows, some paroxysmal disasters, such as flood, can do a great damage in short time. Timely, accurate, and fast acquisition of sufficient disaster information is the prerequisite facing with disaster emergency. Due to UAV's superiority in acquiring disaster data, UAV, a rising remote sensed data has gradually become the first choice for departments of disaster prevention and mitigation to collect the disaster information at first hand. In this paper, a novel and fast strategy is proposed for registering and mosaicing UAV data. Firstly, the original images will not be zoomed in to be 2 times larger ones at the initial course of SIFT operator, and the total number of the pyramid octaves in scale space is reduced to speed up the matching process; sequentially, RANSAC(Random Sample Consensus) is used to eliminate the mismatching tie points. Then, bundle adjustment is introduced to solve all of the camera geometrical calibration parameters jointly. Finally, the best seamline searching strategy based on dynamic schedule is applied to solve the dodging problem arose by aeroplane's side-looking. Beside, a weighted fusion estimation algorithm is employed to eliminate the "fusion ghost" phenomenon.
\end{abstract}

\section{INTRODUCTION}

Overall comprehension of disaster information (for example disaster scale, disaster loss rate, etc.) is crucial for disaster relief and rescue. Remote sensed platforms in aerospace (especially UAV(Unmanned Aerial Vehicle)) have the following advantages over other platforms in taking remote sensing photos of areas in disaster. Firstly, it is less affected by weather, more flexible for operating, and in favour of recycling implementation. Secondly, the images captured from UAV have good characteristics in timeliness and pertinence, as well as large image resolution. Thirdly, the platform performs to have superiority in high reliability, simple structure and easy maintenance, and does not occupy much space. UAV remote sensing data has gradually become the first choice for departments of disaster prevention and mitigation to collect the first-hand disaster information. However, due to the limits of the aerial imaging system, general photographic cameras can only capture scenes from a local area rather than the global distribution in disaster. Therefore, it is necessary to compose all collected UAV images detailed in disaster area into a panorama, in order to fulfil expert's requirement in making decision under emergency condition.

Automatic mosaicing UAV data is a process that makes numbers of UAV images join together into a panoramic, which could cover a lot of scopes. Image mosaic technology is more mature in worldwide excellently, it has developed a wonderful theoretical system especially for panoramic image mosaicing which has an extensive research literature. Nevertheless, there are several commercial offerings encountering with the biggest barrier dues to undeveloped digital cameras at times. Here are some representative researches, in 1975, Kuglin and Hines proposed phase correlation method[(C Kuglin and D Hiness,
1975). While Reddy and Chatterji proposed another method based on fast Fourier transform(FFT-based) in the following second decade(B Srinivasa Reddy and B N Chatterji, 1996). In 1996, Richard Szeliski, who engaged in Microsoft Research, investigated a panorama image mosaic model based on the movement of projection transformation with eight parameters(Richard Szeliski, 1996). M.Irani and S.Peleg utilized the super resolution recovery technology to solve the phenomenon of "fusion ghost"( M.Irani and S.Peleg, 1991). The paper first employ SIFT as operator to extract feature points during primary matching course, following with RANSAC algorithm to eliminate the mismatching feature points, at last, a resolution fusion algorithm was used to make the details among mosaic images be not assimilated, and can ensure the low frequency fusion of background information(M Brown and D G Lowe, 2003). Fortunately, the SIFT algorithm was proposed by David G. Lowe is the most classic and mature SIFT algorithm at present. As his great effort, this favourable descriptor was gradually improved with some formed stitching methods in many manuscripts in the following five years, which partly due to M. Brown's contribution(David G Lowe, 2004). In 2007, Matthew Brown and David G. Lowe gave more details about principle of automatic target identification even under condition that images possess much noise or be in disorder sequence. Simultaneously, the steps of image alignment were also introduced to expand their previous work(Matthew Brow and David G Lowe, 2007). The researches on image mosaic technology were started later in China. Though there has a large gap while compared with the foreign counterparts, many domestic researchers also have made many favourable researches on image mosaic technology. For example, in 1997, Wang established the similarity measurement between template image and target image by using Sequential Similarity Detection Algorithm(SSDA) and Normalized Product Relation

\footnotetext{
* Corresponding author. Ming LI, lisouming@whu.edu.cn
} 
Algorithm, and realized Stochastic Optimization by using Simulated Annealing Algorithm to rapid looking for the best points(Wang and $\mathrm{Wu}, 1997$ ). In 2005, Hou investigated a fast image automatic matching algorithm to resolve the defects of image matching based on similarity in gray level(Hou and Guo, 2005). Fang puts forward an image mosaic algorithm for the problem of "ghost" and exposure difference by using previous image mosaic operator(Fang, Pan and Xu, 2003). In 2009, Zhang proposed an automatic selection principle of DOM's enchase line based on ant colony algorithm, which realizes automatic seamless mosaic with DOM(Digital Orthophoto Map) in surveying and mapping(Zhang, Sun and Zhang, 2009).

Although many successful applications on image mosaic were carried out in last decades, there are still some barriers in the application of UAV images concerning with disaster emergency: firstly, generating DOM from mosaicing UAV images would cost much time, since UAV images are generally in high resolution and cover large area, which may increases computation expense and memory cost while conducting registration process by computer, secondly, as a popular feature descriptor, SIFT(Scale Invariant Feature Transform) appeals to achieve high matching precision, but the convoluted calculation through various scales makes it over burdened in aligning a set of images. Thus, other presented matching algorithms gain lower matching precision though with higher efficiency than SIFT. So it is much troublesome to balance efficiency and effectiveness over a registration task, thirdly, the phenomenon named as "fusion ghost" may emerge when there are objects in large-range motion during the time interval while capturing two adjacent images. Consequentially, it is substantially meaningful to apply UAV images to disaster emergency and to have a research on affection from both aspects of efficiency and effectiveness at the registration course. Besides, a novel workflow including fast rectification and accurate stitching is introduced here to satisfy the demand in disaster emergency.

\section{METHODOLOGY}

\subsection{Optimization of SIFT Feature Extraction}

In 1999, SIFT operator was first put forward by David G. Lowe at British Columbia university, and it was summarized and improved in 2004. Then, Y. Ke amended the local of algorithm, he used the PCA instead of the histogram to form its descriptor. SIFT operator looking for extreme value point in scale space, and extract position, dimension and rotation invariants, the operator has some stability, uniqueness, quantity and extensibility. SIFT feature extraction is generally classified into four steps: detect extreme value in scale space, tie point positioning, dominant orientation assignment, and descriptor generation. In order to guarantee the stability of SIFT algorithm feature extraction and ensure sufficient number of feature points, usually, many papers amplifier the image as 2 times as its original size when building its corresponding scale space, and each octave is suggested to build 5 pyramid levels at different Gaussian scales. In this process, the time cost of construction image pyramid is more than half of the time of SIFT feature extraction.

Since obtaining the adjacent UAV images spend less time and the flight attitude of UAV are relatively stable, there usually won't appear to have very large translation, rotation, scaling among the adjacent images, that is the image size has a small change. In order to obtain enough accurate feature points and accelerate matching process, we need to consider two factors: $\Phi$ shorten matching time; $\varnothing$ achieve stabile feature extraction, it is not advisable to gain a lot of mismatch tie points as shorten the time of feature extraction. Figure 1(a) and 1(b) shown that are the contrast of operation time and numbers of feature points under different SIFT improve operators.

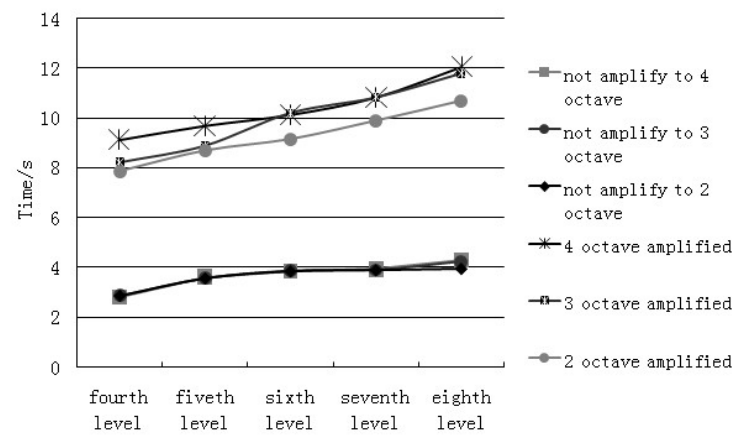

(a)

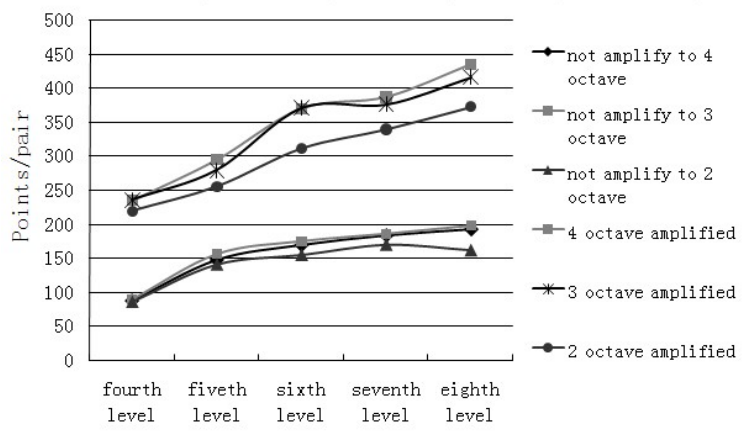

(b)

Figure 1. (a) Contrast the operation time of different SIFT Operators, (b) Contrast numbers of feature points of different SIFT operators.

We can see from the stability experimental results above, when the image is not to amplified 2 times to build scale space at the first level of the first octave, and the octave number is 2 , the level number in each octave is 4 , we can shorten about $70 \%$ time of SIFT feature extraction, and the feature points are much more than points the least-square need for soluting the imaging movement model parameters, the number of octaves has little effect on time and stability of SIFT feature extraction, and will not lead to abundant mismatched feature points. So, the following experiments of this paper will adopt this method mentioned above.

\subsection{RANSAC Operator}

There is still a certain number of mismatching points after SIFT key point matching, it's necessary to eliminate the mismatching points for precision of image registration, this paper used RANSAC(Random Sample Consensus) operator to solve the problem. RANSAC operator is a parameter estimation method with robustness, RANSAC operator's basic idea is: first, design a certain target function according to specific problems; second, extract minimum points repeatedly to estimate the initial value of parameters in that target function, and then use the initial value to divide all the data into "interior point(inlier)" and "out point(outlier)"; finally, recompute and re-estimate the parameters of the function by all the interior points. Figure 2 shown that the mosaic effect of before and after the mismatch points elimination. 


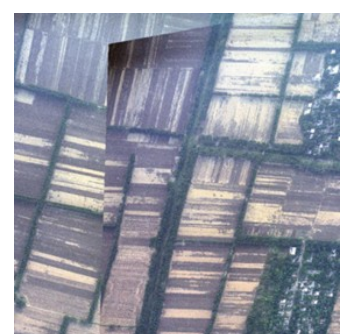

(a)

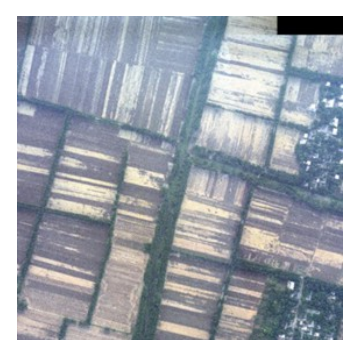

(b)
Figure 2. (a) Mosaic effect before the mismatch points elimination, (b) mosaic effect after the mismatch points elimination.

\subsection{Transform Optimization}

As a perfect panoramic stitching, it requires the minimum gray error in images overlap area after transform correction and seamlessly image.

$$
E=\sum_{i}\left(I\left(x^{\prime}, y^{\prime}\right)-I(x, y)\right)^{2}=\sum_{i} e_{i}^{2} .
$$

In 1996, Richard Szeliski expounded the use of LevenbergMarquardt method in his paper: "Video Mosaics for Virtual Environments". He said that we can use the LevenbergMarquardt iterative nonlinear minimum algorithm to optimize the rotation matrix, and by this way to realize minimum mosaic gray error. It also satisfies the idea of the minimum visual deviation in computer vision. Following is the rotation matrix of 8 parameters rotation transformation model

$$
A=\left[\begin{array}{ccc}
m_{0} & m_{1} & m_{2} \\
m_{3} & m_{4} & m_{5} \\
m_{6} & m_{7} & 1
\end{array}\right],
$$

its rotation matrix has 8 parameters, so we need to seek the partial derivative of every parameter the gray error $e$ by the Levenberg-Marquardt method, the formulas are as follows:

$$
\begin{aligned}
& \frac{\partial e_{i}}{\partial m_{0}}=\frac{x_{i}}{D_{i}} \frac{\partial I}{\partial x^{\prime}}, \frac{\partial e_{i}}{\partial m_{1}}=\frac{y_{i}}{D_{i}} \frac{\partial I}{\partial x^{\prime}}, \frac{\partial e_{i}}{\partial m_{2}}=\frac{1}{D_{i}} \frac{\partial I}{\partial x^{\prime}}, \\
& \frac{\partial e_{i}}{\partial m_{3}}=\frac{x_{i}}{D_{i}} \frac{\partial I}{\partial y^{\prime}}, \frac{\partial e_{i}}{\partial m_{4}}=\frac{y_{i}}{D_{i}} \frac{\partial I}{\partial y^{\prime}}, \frac{\partial e_{i}}{\partial m_{\mathrm{s}}}=\frac{1}{D_{i}} \frac{\partial I}{\partial y^{\prime}}, \\
& \frac{\partial e_{i}}{\partial m_{6}}=-\frac{x_{i}}{D_{i}}\left(x^{\prime} \frac{\partial I}{\partial x^{\prime}}+y^{\prime} \frac{\partial I}{\partial y^{\prime}}, \frac{\partial e_{i}}{\partial m_{7}}=-\frac{y_{i}}{D_{i}}\left(x^{\prime} \frac{\partial I}{\partial x^{\prime}}+y^{\prime} \frac{\partial I}{\partial y^{\prime}}\right) .\right.
\end{aligned}
$$

$\mathrm{D}_{i}=m_{6} x+m_{7} y+1$ is the denominator of 8 parameters transformation equation, $\frac{\partial I}{\partial x^{\prime}}$ and $\frac{\partial I}{\partial y^{\prime}}$ are the gray gradients of the point, they are replaced with difference approximatively, as follows:

$$
\begin{aligned}
& \frac{\partial I}{\partial x^{\prime}}=\frac{1}{2}\left(I\left(x^{\prime}+1, y^{\prime}\right)-I\left(x^{\prime}-1, y^{\prime}\right)\right) \\
& \frac{\partial I}{\partial y^{\prime}}=\frac{1}{2}\left(I\left(x^{\prime}, y^{\prime}+1\right)-I\left(x^{\prime}, y^{\prime}-1\right)\right) .
\end{aligned}
$$

Then, calculate the approximate $\operatorname{Hessian} \operatorname{matrix}(A)$ and weighted gradient vector $(b)$, Hessian matrix $(A)$ and gradient $\operatorname{vector}(b)$ are as follows:

$$
\begin{aligned}
& A=\left[a_{k l}\right]=\sum_{i} \frac{\partial e_{i}}{\partial m_{k}} \bullet \frac{\partial e_{i}}{\partial m_{l}} \\
& b=\left[b_{k}\right]=-\sum_{i} e_{i} \frac{\partial e_{l}}{\partial m_{k}},(k, l=1,2 \mathrm{~L} 8) .
\end{aligned}
$$

" $A$ " is optimization updated by the increment $\Delta m=(A+\lambda I)^{-1} b, \lambda$ is a stability factor changed with time, Levenberg-Marquardt method can converge in a few times of iteration and get the answers.

\subsection{Bundle Adjustment}

Given a set of geometrically consistent matches between the images, we use bundle adjustment to solve for all of the camera parameters jointly. This is an essential step as concatenation of pairwise homography would cause accumulated errors and disregard multiple constraints between images e.g. that the ends of a panorama should join up. Images are added to the bundle adjuster one by one, with the best matching image (maximum number of matches) being added at each step. The new image is initialized with the same rotation and focal length as the image to which it best matches. Then the parameters are updated using Levenberg-Marquardt(Matthew Brow and David G Lowe, 2007). The objective function we use is a robust sum squared projection error. That is, each feature is projected into all the images in which it matches, and the sum of squared image distances is minimized with respect to the camera parameters.

\subsection{Precision Evalution}

Geometric registration error is an important indicator to measure precision of registration algorithm. If the registration error is too large, the subsequent steps of images mosaic will be unable to go on. The next experiment select 8 parameters projection transform model as the registration model, and utilize optimization algorithm of SIFT feature extraction in this paper and RANSAC operator to eliminate the mismatching feature points, we get 30 pairs of feature points. Table 1 represents the image registration single point RMS value obtained from RMS formula, the average geometric registration error we get through totalRMS formula is 1.96798 , and the precision of the experiment calibration error meets demand of the UAV image mosaic requirements in emergency situation.

\subsection{Image Mosaic Way}

UAV images can be mosaiced into a panoramic only when they are transformed to the same coordinate system, Transfer approach of matching coordinate system cause the precision and shape of mosaiced image. This paper adopts the way of frame to frame according to a contrast and analysis of four stitching models: frame to frame, frame to stitching images, stitching images to frame, stitching images to stitching images; and through comprehensive consideration of complexity, calculated amount and accumulative error in the process. At first, the way of frame to frame select a UAV image as a reference image by observing some criterions; then, transform the other images into the coordinate system of the reference image, the coordinate system is fixed in the whole joining together process. The selection of reference image can be an image in UAV images, also a virtual reference coordinate system is okay. All we need to do is transforming images to the same coordinate system. Shown in figure 4 is the schematic plot of image mosaic by frame to frame:

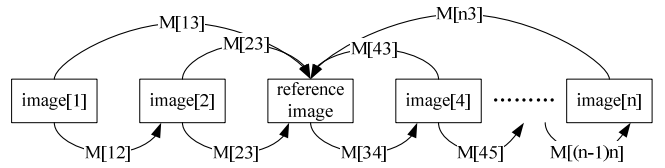

Figure 4. Schematic plot of image mosaic by frame to frame

\subsection{Analysis of Reprojection Method}

Through the method mentioned above, after calculating the imaging movement model parameters of the sequence images 
International Archives of the Photogrammetry, Remote Sensing and Spatial Information Sciences, Volume XXXIX-B6, 2012 XXII ISPRS Congress, 25 August - 01 September 2012, Melbourne, Australia

\begin{tabular}{cccccc}
\hline & \multicolumn{2}{c|}{ Control points } & & \multicolumn{2}{c}{ Control points } \\
\hline RMS & Left $(\mathrm{X} / \mathrm{Y})$ & Right $(\mathrm{X} / \mathrm{Y})$ & RMS & Left $(\mathrm{X} / \mathrm{Y})$ & Right $(\mathrm{X} / \mathrm{Y})$ \\
\hline 2.17318 & $408 / 336$ & $83 / 426$ & 2.01241 & $423 / 423$ & $332 / 531$ \\
2.06731 & $389 / 424$ & $268 / 532$ & 2.00217 & $431 / 486$ & $135 / 476$ \\
2.14382 & $424 / 525$ & $100 / 611$ & 1.97590 & $424 / 381$ & $128 / 470$ \\
2.16912 & $436 / 24$ & $109 / 25$ & 1.94325 & $329 / 597$ & $233 / 704$ \\
2.13252 & $403 / 354$ & $77 / 443$ & 1.93284 & $423 / 24$ & $128 / 16$ \\
2.11049 & $329 / 631$ & $202 / 737$ & 1.87842 & $271 / 606$ & $175 / 713$ \\
2.14393 & $215 / 71$ & $96 / 53$ & 1.83275 & $375 / 45$ & $79 / 37$ \\
2.18621 & $221 / 332$ & $96 / 436$ & 1.82163 & $358 / 42$ & $63 / 32$ \\
2.18446 & $181 / 45$ & $61 / 51$ & 1.80383 & $147 / 178$ & $57 / 283$ \\
2.02438 & $435 / 401$ & $313 / 518$ & 1.80123 & $156 / 135$ & $65 / 240$ \\
2.03494 & $268 / 359$ & $145 / 464$ & 1.78286 & $387 / 69$ & $300 / 105$ \\
2.13583 & $378 / 529$ & $252 / 632$ & 1.73437 & $401 / 554$ & $99 / 641$ \\
2.04274 & $149 / 366$ & $26 / 470$ & 1.73198 & $194 / 515$ & $99 / 621$ \\
2.19662 & $269 / 619$ & $142 / 726$ & 1.71265 & $261 / 627$ & $166 / 734$ \\
2.15523 & $414 / 516$ & $287 / 623$ & 1.65275 & $379 / 58$ & $84 / 47$ \\
\hline
\end{tabular}

Table 1. RMS results of single point.

\begin{tabular}{lllllll}
\hline $\begin{array}{l}\text { Image } \\
\text { sequence }\end{array}$ & $\begin{array}{l}\text { Feature points } \\
\text { rough match }\end{array}$ & $\begin{array}{l}\text { Numbers of } \\
\text { interior points }\end{array}$ & RANSAC & totalRMS & Match time/s & Mosaic time/s \\
\hline $1-2$ & 80 & 78 & 1.85358 & 2.59897 & 4.10320 \\
$2-3$ & 81 & 80 & 1.93049 & 2.68172 & 8.99765 \\
$3-4$ & 78 & 73 & 1.84173 & 2.79301 & 15.8359 \\
$4-5$ & 79 & 75 & 1.80460 & 2.80215 & 20.9801 \\
$5-6$ & 90 & 89 & 1.87805 & 2.88417 & 28.9662 \\
$6-7$ & 86 & 84 & 1.93290 & 2.90121 & 31.9813 \\
$7-8$ & 96 & 93 & 1.92133 & 2.88064 & 38.0137 \\
$8-9$ & 90 & 88 & 2.01062 & 2.90892 & 43.8193 \\
$9-10$ & 88 & 86 & 1.82356 & 2.94401 & 51.0013 \\
$10-11$ & 95 & 93 & 2.00540 & 2.98610 & 56.9936 \\
$11-12$ & 93 & 91 & 1.90035 & 2.89867 & 66.0798 \\
\hline
\end{tabular}

Table 2. Index statistics of the experiment

frame to frame, select reference images, and re-project other images into a unified two-dimensional surface to mosaic. Considering the troublesome of re-projecting the main spherical manifold in two-dimensional surface and cube manifold, it was rarely used. By comparing the cylinder manifolds and plane manifold, the later one is simpler and commonly used. After image re-projection the straight line remains the same, closer to the reality of object shape, and the experimental plot image perspective span is tiny, that is why we use the plane manifold.

\subsection{Image Fusion Method Selection}

Directly overlay images after registration will appear obvious seamline, adverse visual feelings and low-quality stitching. Many factors that affect the quality of image stitching, including image matching error, correction model error, exposure differences, and white balance, Shown in Figure $5(\mathrm{a})$ and $5(\mathrm{~b})$ are image quality problems caused by matching errors and exposure differences exist for stitching images.

It can be seen from Figure 5(a), 5(b) that images appear problems of house dislocation and oversize colour difference problems, and images are stitched together forming a seam. In order to solve these problems, it usually considers image fusion strategy to eliminate the impact of these factors, and the most commonly used is the direct average method and weighted fusion method. There will be discontinuous phenomenon at the seamline by using direct average method, but the weighted fusion can overlapping regional gray gradient, eliminate the seam vision, if there are registration errors between two images, simply using weighted fusion would give rise to the phenomenon of fusion ghosting, without achieving a perfect seamless effect. Figure 6 is a weighted fusion of two image after joining together, from it can see although the seamline got a very good processing, but the location of marked red circle in serious "fusion ghost" phenomenon.

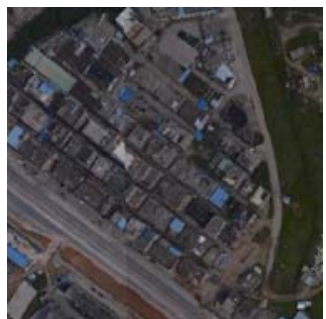

(a)

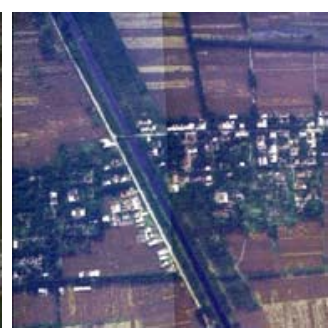

(b)
Figure 5. (a) The problem caused by matching errors, (b) the problem caused by exposure differences.

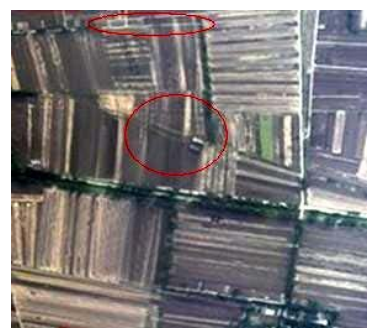

Figure 6. Fusion ghost. 


\subsection{The Best Seamline Search Based on Dynamic Programming}

In 1998, Duplaquet proposed a best seam search criteria.

$$
E(x, y)=E_{\text {color }}(x, y)^{2}+E_{\text {geomerty }}(x, y) \text {. }
$$

In this formula: $E_{\text {color }}$ represents the gray value difference, $E_{\text {geometry }}$ - the structural difference of all overlaps pixels between two images. And $E_{\text {geometry }}$ is completed by modifying Sobel operator. But when using Sobel operator to calculate the gradient does not take into account the similarity of the pixels surrounding structures, and therefore not able to find the best seamline. Considering the difference of the four edge pixels as the evaluation criteria to measure the structural similarity of the pixel geometry, we use the new gradient calculation template in this paper. And that is the $s_{x}, s_{y}$ new gradient templates in the $x$ and $y$ direction of vector.

$$
s_{x}=\left[\begin{array}{lll}
-2 & 0 & 2 \\
-1 & 0 & 1 \\
-2 & 0 & 2
\end{array}\right] \text { and } s_{y}=\left[\begin{array}{ccc}
-2 & -1 & -2 \\
0 & 0 & 0 \\
2 & 1 & 2
\end{array}\right]
$$

If $f_{1}$ and $f_{2}$ for any two of the original image for joining together, so $E_{\text {geometry }}$ calculation formula is as follows:

$$
E_{\text {geomerty }}=\operatorname{Diff}\left(f_{1}(x, y), f_{2}(x, y)\right) .
$$

Diff calculation is first calculate gradient difference of $f_{1}$ and $f_{2}$ respectively in $x$ and $y$ directions, then multiply together difference of two gradient to achieve the final result. In order to solve the problems of incompletely eliminate seam and "fusion ghost" by simply using weighted average, this paper puts forward the best seamline search strategies based on the dynamic planning, and combined weighted provided to solve the above problems.

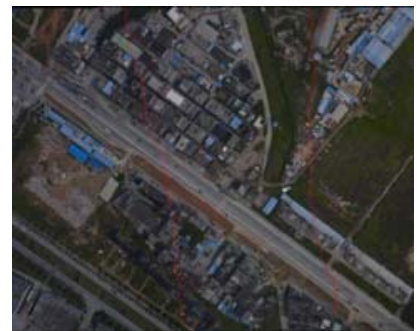

Figure 7. The diagram of the best seamline search based on dynamic programming.

The red line in Figure 7 is the results of best seamlines by the best seamline search strategy based on dynamic programming. We can see from the above diagram that the seamline completely bypass houses, and the gray difference is very small on both sides of seamline. Basicly, the strategy ensure Its quality meet requirements of seamless mosaic along the seamlines on the UAV images, and it is effectively to get rid of "fusion ghost". Because of the colour difference is so large of adjacent images, by using the strategy is mentioned above, that we only can not see the phenomenon of dislocation, there is still has colour difference. In order to resolve this situation that is a fixed colour difference along the seamline, we can select $n$ (the value of $n$ should not be too big) pixels both sides along seamlines respectively, then, do weighted average to pixels in the eclosion area for realizing the smooth transitions to grayscale.

\section{THE EXPEREMENT SYSTEM DESIGN AND RESULTS ANALYSIS}

\subsection{Image Mosaicing System}

This paper puts forward a new automatic UAV image mosaicing system. The development of this system is based on three steps: candidate feature extraction and matching, geotransform model estimation and optimization, and image fusion. The main modules of this system include: module of feature extraction and matching, module of gross error elimination (by RANSAC algorithm), module of model estimation, module of seamline tracking, module of eclosion process, etc. In this system, module of feature extraction and matching is mainly based on SIFT operator, its matching course is accelerated by k$\mathrm{d}$ tree, Beside, original image is not amplified to be 2 times when building scale space, solution of the transformational model for optimization of model parameters by the method of transformation model with 8 parameters, module of gross error elimination based on RANSAC operator can effectively detect and rectify the gross error, bundle adjustment is used to calculate the camera calibration parameters, module of seamline tracking is based on dynamic programming, while image fusion adopts the weighted eclosion strategy. Experiments show that these method and strategy can make image matching be more efficient, and the grayscale of panoramic is smoothly transitioned. The core modules along with algorithms introduced above were all programmed in $\mathrm{C}++$ language, GDAL and GSL were also utilized as additional libraries to ease the programming progress. Figure 8 shows the flowchart of UAV image mosaic strategy working on through all experiments detailed as below.

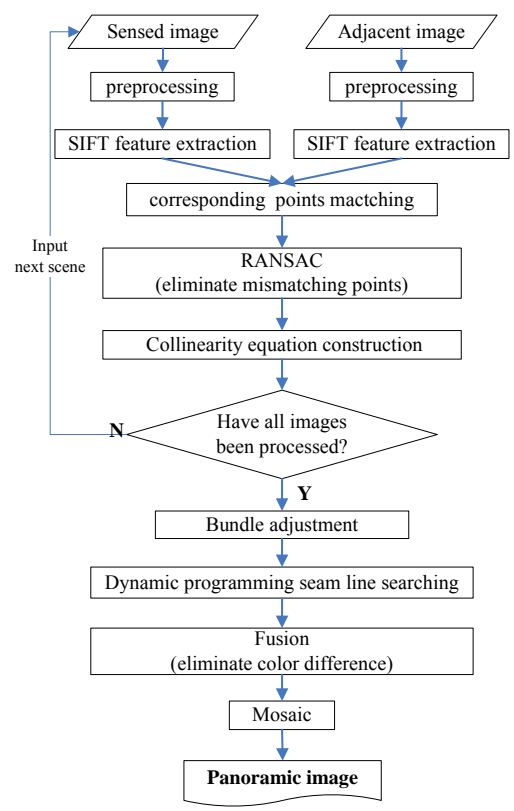

Figure 8. Flowchart of the UAV image mosaic.

\subsection{Experimental Environment and Data}

The experiment of this paper is completed by a DELL desktop computer, it has a Inter pentium(R) Dual-core CPU, $2.8 \mathrm{GHz}$, $2 \mathrm{G}$ RAM. The size of experimental images is $680 \times 912$. These images are taken by UAV in the suburb area of PingShan, the photography flying height is about $1000 \mathrm{~m}$, ranks number of image is $5413 \times 7218$, the rate of head overlap is $68 \%$, and the rate of image sideslip is $42 \%$. It is difficult to match accurately 
because of the photographing area is full of houses, roads, and other buildings.

\subsection{Experimental Results Analysis}

Through the mosaic experiment with 12 images from the same air strip in PingShan area, we can get 5 indexes include feature points of rough matching, numbers of RANSAC interior points, totalRMS, matching time, time for mosaic, We put the statistical data in table 2 . We can see from the table 2 that the UAV panorama has a high accuracy, and doesn't take much time. That means UAV image mosaic system is able to meet the requirements for disaster emergencies and can be used in cities with paroxysmal disasters. Figure 9 is the mosaic rendering with 12 images in the first air strip, Figure 10 is the mosaic rendering with 35 images from three air strips in experimental area.

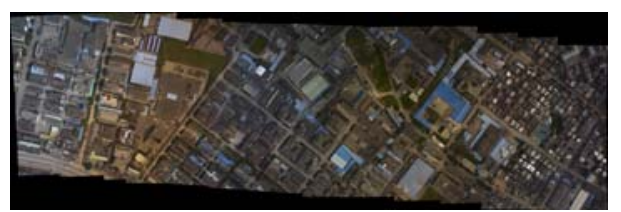

Figure 9. Mosaic rendering with 12 images in the first air strip.

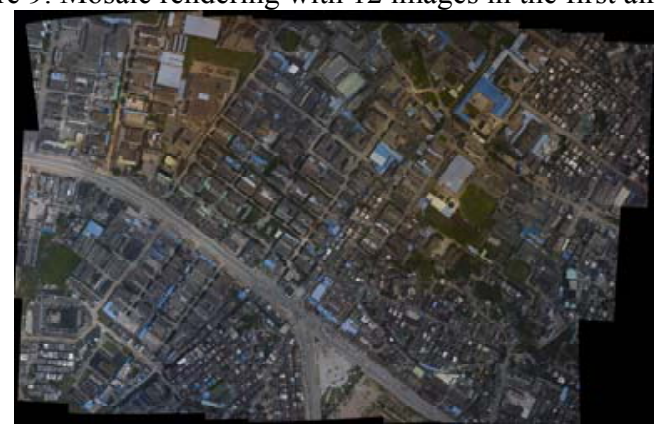

Figure 10. Mosaic rendering with 35 images from three air strips.

According to the experiments mentioned above, the mosaic technical scheme of UAV image is effective and worth spreading. By using it, we can accelerate the image processing, avoid the surface feature dislocation from matching precision, eliminate the "fusion ghost" phenomenon from using simple weighted fusion as existence matching error exists, realize harmonious tone of many mosaic images, and weaken the seamlines.

\section{CONCLUSION}

Analysis of the UAV images' mosaic experimental results in urban residential areas of Pingshan in the city of Wuhan, China, this paper presents a new system for aforementioned fully automatic panorama stitching, which is little interactive with manual configuration and operation, and high efficient on registration and mosaicing process. The manufactured panorama images appear to achieve acceptable accuracy and be robust to unstable camera poses. Meanwhile, noisy and illumination change are eliminated to some degree. Under this excellent application framework, Experiments show that the proposed UAV images' mosaicing strategy could provide fundamental and timely technology support for acquiring firsthand disaster information for disaster emergency and relief.

\section{ACKNOWLEDGEMENTS}

This paper supported by "the Fundamental Research Funds for the Central Universities (No.2012213020206)”.

\section{REFERENCE}

C Kuglin and D Hines, 1975. The phase correlation image alignment method. In Proc IEEE Conference on Cybernetics and Society, pp. 163-165 .

B Srinivasa Reddy and B N Chatterji, 1996. An FFT-based technique for translation, rotation, and scale-invariant image registration. IEEE Transactions on Image Processing. 8(5), pp. 1266-1271.

Richard Szeliski, 1996. Video mosaics for virtual environments. IEEE Computer Graphics and Applications, 16(2, pp. 22-30.

M Irani S Peleg, 1991. Improving Resolution by Image Registration. Graph Models Image Process . 53(3), pp. 231-239.

M Brown and D G Lowe, 2003. Recognising Panoramas .In Proceedings of the 9th IEEE International Conference on Computer Vision, pp. 1218-1225.

DAVID G LOWE, 2004. Distinctive Image Features from Scale-Invariant Keypoints. Computer Science Department , 60(2), pp. 91-110.

Matthew Brown and David G Lowe, 2007. Automatic Panoramic Image Stitching using Invariant Features. International Journal of Computer Vision . 74(1), pp. 59-73.

Xiao Rui, Xincai Wu, 1997. Remote Images Automatic Registration Method, Journal of Image and Graphics,2(10), pp. 735-739.

Shuwei Hou, Baolong Guo, 2005. A fast algorithm of image to be automatic joining together,Computer Engineering, 31(15), pp. $70-72$.

Xianyong Fang, Zhigen Pan, 2003. Image mosaicing algorithm, Computer Aided Design and Graphics of Journal, 15(11), pp. 1362-1366.

Jianqing Zhang, Mingwei Sun, Zuxun Zhang, 2009. Based on ant colony algorithm is like set automatic selection of projective line, Geomatics and Information Science of Wuhan University, 34(6), pp. 675-678. 